Cómo citar este artículo: Trejos-Salazar, D. F., Duque-Hurtado, P. L., Montoya-Restrepo, L. A., \& Montoya-Restrepo, I. A. (2021). Neuroeconomía: Una revisión basada en técnicas de mapeo científico. Rev.investig.desarro.innov., 11 (2), 243-260.

\title{
Neuroeconomía: una revisión basada en técnicas de mapeo científico
}

\section{Neuroeconomics: a review based on scientific mapping techniques}

\section{Damiand Felipe Trejos-Salazar ${ }^{1}$ Pedro Luis Duque-Hurtado² Luz Alexandra Montoya-Restrepo ${ }^{3}$ Iván Alonso Montoya-Restrepo ${ }^{4}$}

Recibido: abril 13 de 2020

Aceptado: septiembre 15 de 2020

\section{Resumen}

La neuroeconomía es un campo multidisciplinar, que articula los conocimientos de áreas como la Economía, la psicología y la neurociencia, y que estudia el comportamiento cerebral en la toma de decisiones. A través de una revisión de literatura, se presenta la evolución de la investigación en neuroeconomía. Para ello, se emplean técnicas de mapeo científico, apoyadas en herramientas bibliométricas. La búsqueda, se realizó en las bases de datos WoS y Scopus, y la información obtenida fue procesada con las herramientas Bibliometrix y Gephi. Los documentos se clasificaron según su relevancia, en tres categorías: clásicos, estructurales y actuales. Luego, a través de un análisis de co-citaciones y clusterización, se identificaron y analizaron cinco líneas o corrientes de investigación en el área, a saber: elecciones económicas, elección social, consideraciones sobre la neuroeconomía, neurociencia del consumidor y comportamiento y estímulo cerebral. Se concluye con la necesidad de encontrar y estandarizar una metodología de investigación, en la que converjan los criterios para fortalecer los resultados de las investigaciones realizadas, ya que no se pueden desconocer las limitaciones de las metodologías actuales.

Palabras clave: neuroeconomía, toma de decisiones, elección social, recompensa.

\begin{abstract}
Neuroeconomics is a multidisciplinary field, which articulates the knowledge of areas such as economics, psychology and neuroscience, and which studies brain behavior in decisionmaking. Through a literature review, the evolution of research in neuroeconomics is presented. For this, scientific mapping techniques are used, supported by bibliometric tools. The search was carried out in the WoS and Scopus databases, and the information obtained was processed with the Bibliometrix and Gephi tools. The documents were classified according to their relevance, into three categories: classic, structural and current. Then, through an analysis of co-citations and clustering, five lines or currents of research in the area were identified and analyzed, namely: economic choices, social choice, considerations on neuroeconomics, consumer neuroscience and behavior and brain stimulation. It concludes with the need to find and standardize a research methodology, in which the criteria converge to strengthen the results of the research carried out, since the limitations of current methodologies cannot be ignored.
\end{abstract}

Keywords: neuroeconomics, decision making, social choice, reward.

1 Administrador de Empresas, Magíster en Administración, Universidad de Caldas, Manizales, Colombia. E-mail: damiand.trejos@ ucaldas.edu.co

ORCID: https://orcid.org/0000-0002-3207-5432

2 Administrador de Empresas, Magíster en Administración, Universidad Católica Luis Amigó, Manizales, Colombia. E-mail: pedro. duquehu@amigo.edu.co

ORCID: https://orcid.org/0000-0003-4950-8262

3 Administradora de Empresas, Doctora en Ciencias Económicas, Universidad Nacional de Colombia, Medellín, Colombia. E-mail: lamontoyar@unal.edu.co

ORCID: https://orcid.org/0000-0002-4896-1615

4 Administrador de Empresas, Doctor en Ciencias Económicas, Universidad Nacional de Colombia, Medellín, Colombia. E-mail: iamontoyar@unal.edu.co

ORCID: https://orcid.org/0000-0003-0959-3466 


\section{Introducción}

La neuroeconomía se ha desarrollado como una nueva rama del conocimiento, que ha posibilitado un crecimiento investigativo relacionando la neurociencia y la economía (Ramírez, 2010) y que además, ha permitido fortalecer los temas de gestión ya que también permite comprender la toma de decisiones en ámbitos económicos y financieros (McKiernan, 2017). Es a partir de los estímulos neuronales, que los científicos pueden conocer mucho mejor los procesos de elección.

Algunas investigaciones demuestran, además, la relación de elementos de la psicología con la neuroeconomía, permitiendo interpretar conductas en los procesos de elección de las personas en situaciones controladas, para ser contrastadas posteriormente con las teorías económicas (Krause \& Lindemann, 2014; Smith et al., 2004). La evaluación del cerebro al momento de tomar decisiones, se convierte en un paso importante en la comprensión de las elecciones, ya que busca identificar procesos cognitivos y físicos en la conducta humana (D'Angiulli et al., 2012; Trepel et al., 2005). Por consiguiente, es necesario investigar en neuroeconomía para entender la conducta humana en la toma de decisiones.

Se evidencian algunas investigaciones de tipo revisión sobre el tema. Glimcher y Rustichini (2004), realizan una revisión del desarrollo teórico de la neuroeconomía, desde la perspectiva conductual y biológica. Rangel et al. (2008), presentan un marco para investigar diferentes aspectos de la neurobiología en la toma de decisiones. Yu y Zhou (2007), efectúan una revisión de la literatura, pero su enfoque radica en análisis de resultados cerebrales y la relación con teorías y métodos de la economía. Westbrook y Braver (2015), analizan algunas teorías de la psicología y la neurociencia asociadas con el esfuerzo cognitivo, y describen los beneficios de la investigación en neuroeconomía. Sin embargo, a la fecha no se ha identificado una revisión que emplee el análisis de redes y técnicas de mapeo científico, para estudiar la evolución de la neuroeconomía.
En vista de lo anterior, este documento pretende realizar una revisión de literatura de neuroeconomía basado en técnicas de mapeo científico y herramientas bibliométricas, como Bibliometrix y Gephi, para identificar perspectivas o corrientes de investigación sobre el tema. El documento se estructura en tres secciones, iniciando con la metodología usada para elaborar la revisión. Luego, se expone el tema a través de las herramientas bibliométricas, la evolución y perspectivas del tema $y$, finalmente, se presentan las conclusiones, limitaciones y recomendaciones para investigaciones de este tipo.

\section{Metodología}

La metodología se desarrolló en tres etapas. La primera consistió en realizar un análisis de mapeo científico, basado en los métodos bibliométricos sugeridos por Zupic y Čater (2015), usando la herramienta Bibliometrix (Aria \& Cuccurullo, 2017), la cual ha sido empleada y validada en múltiples investigaciones (Addor \& Melsen, 2019; Almeida \& de Paula 2019; Bond \& Buntins, 2018; Medina et al., 2018). Posteriormente, se realizó un análisis cronológico utilizando la metáfora de árbol, en la que se identificaron las subáreas del tema mediante las co-citaciones. A continuación, se detalla el procedimiento empleado.

La importancia de la neuroeconomía, se determinó utilizando tres métricas: producción científica anual, relevancia de las revistas y visibilidad de los autores. La búsqueda de los documentos se realizó en las bases de datos Scopus y Web of Science (WoS). Los criterios de consulta, fueron: "Neuroeconomy*" OR "Neuroeconomic*", y estas palabras se tuvieron en cuenta en: los temas, títulos, resúmenes y palabras clave. El periodo de tiempo considerado fue 2005 a 2019, y la consulta se realizó el 15 de febrero de 2020. Se encontraron 978 registros en WoS y 1103 en Scopus.

Los resultados fueron analizados empleando la analogía del árbol, propuesta por Robledo et al. (2014), la cual, mediante la selección de documentos y autores identificados en la búsqueda por WoS y Scopus, permite comprender y abor- 
dar un tema específico. El método categoriza como "nodos" a los artículos, y a las conexiones entre los mismos como "aristas". Los criterios de clasificación de los nodos, ubica primero los documentos con mayor citación en la raíz del árbol, identificándolos como hegemónicos. Posteriormente, presenta los documentos que citan la raíz y son citados por las hojas, cuya clasificación es de estructurales. Finalmente, las hojas citan los artículos de la raíz y el tronco, es decir, los demás de la red. La analogía, permite visualizar e interpretar la producción científica. Esta metodología ha sido abordada en investigaciones recientes (Buitrago et al., 2020; Duque \& Cervantes-Cervantes, 2019; Landínez-Martínez et al., 2019; Marín-López et al., 2017; Zuluaga et al., 2016).
En la Figura 1, se presenta un esquema de árbol en el que se identifican los artículos más importantes, la relevancia se determinó mediante la selección de diez de cada zona permitiendo ampliar el panorama sobre los conceptos, las bases, las aproximaciones actuales y la historia de la neuroeconomía. Una vez seleccionados los artículos de mayor interés, se presenta un análisis objetivo del tema y sus perspectivas. Las perspectivas son determinadas mediante el análisis de co-citaciones mediante el algoritmo de clusterización (Blondel et al., 2008) proceso en el que se identifican y analizan los temas que las componen mediante la minería de texto a través de " $R$ ", específicamente con el algoritmo Wordcloud (Ohri, 2013; Robledo-Giraldo et al., 2013).

\begin{tabular}{|c|c|c|c|c|c|c|c|c|c|c|}
\hline & \multicolumn{2}{|c|}{ Perspectiva 1} & \multicolumn{2}{|c|}{ Perspectiva 2} & \multicolumn{2}{|c|}{ Perspectiva 3} & \multicolumn{2}{|c|}{ Perspectiva 4} & \multicolumn{2}{|c|}{ Perspectiva 5} \\
\hline \multirow{5}{*}{$\begin{array}{c}\text { Hojas } \\
\text { Perspectivas }\end{array}$} & \multicolumn{2}{|c|}{ Balasubramani Pp, (2018) } & \multicolumn{2}{|c|}{ Luo Jy, (2015) } & \multicolumn{2}{|c|}{ Fumagalli $R,(2016,2017)$} & \multicolumn{2}{|c|}{ Lee N, (2018) } & \multicolumn{2}{|c|}{ Jarmolowicz Dp, (2016) } \\
\hline & \multicolumn{2}{|c|}{ Domenech P, (2018) } & \multicolumn{2}{|c|}{ Sonuga-Barke Ejs, (2016) } & \multicolumn{2}{|c|}{ Serra D, (2012) } & \multicolumn{2}{|c|}{ Karmarkar Ur, (2019) } & \multicolumn{2}{|c|}{ Regier Ps, (2015) } \\
\hline & \multicolumn{2}{|c|}{ Webb R, (2019) } & \multicolumn{2}{|c|}{ Zald Dh, (2017) } & \multicolumn{2}{|c|}{ Ardalan K, (2018) } & \multicolumn{2}{|c|}{ Sawe N, (2017) } & \multicolumn{2}{|c|}{ Wilson Ag, (2015) } \\
\hline & \multicolumn{2}{|c|}{$\operatorname{Lin} H,(2018)$} & \multicolumn{2}{|c|}{ Krueger F, 2019) } & \multicolumn{2}{|c|}{ Herrmann-Pillath C, (2016) } & \multicolumn{2}{|c|}{ Ramsoy Tz, (2017) } & \multicolumn{2}{|c|}{ Peters J, (2012) } \\
\hline & \multicolumn{2}{|c|}{ Clithero Ja, (2018) } & \multicolumn{2}{|c|}{ Robson Se, (2020) } & \multicolumn{2}{|c|}{ Erixon L, (2015) } & \multicolumn{2}{|c|}{ Yokoyama R, (2014) } & \multicolumn{2}{|c|}{ Pehlivanova M, (2018) } \\
\hline Tronco & $\begin{array}{c}\text { Kenning \& } \\
\text { Plassmann, } \\
(2005)\end{array}$ & $\begin{array}{l}\text { Sanfey, } \\
(2007)\end{array}$ & $\begin{array}{l}\text { Loewenstein } \\
\text { et al., (2008) }\end{array}$ & $\begin{array}{l}\text { Kishida et } \\
\text { al., (2010) }\end{array}$ & $\begin{array}{c}\text { Fehr \& } \\
\text { Rangel, } \\
(2011)\end{array}$ & $\begin{array}{r}\text { Monterosso } \\
\text { et al., (2012) }\end{array}$ & $\begin{array}{c}\text { Delgado \& } \\
\text { Dickerson, } \\
\text { (2012) }\end{array}$ & $\begin{array}{c}\text { C. F. } \\
\text { Camerer, } \\
(2013)\end{array}$ & $\begin{array}{c}\text { Kopton \& } \\
\text { Kenning, } \\
\text { (2014) }\end{array}$ & $\begin{array}{c}\text { Fumagalli, } \\
\text { (2016) }\end{array}$ \\
\hline Raíz & $\begin{array}{c}\text { Kahneman \& } \\
\text { Tversky, } \\
\text { (1979) }\end{array}$ & $\begin{array}{c}\text { Schultz et al., } \\
\text { (1997) }\end{array}$ & $\begin{array}{c}\text { Sanfey et al., } \\
\text { (2003) }\end{array}$ & $\begin{array}{l}\text { McClure, } \\
\text { Laibson, et } \\
\text { al., (2004) }\end{array}$ & $\begin{array}{c}\text { Glimcher \& } \\
\text { Rustichini, } \\
\text { (2004) }\end{array}$ & $\begin{array}{r}\text { C. Camerer } \\
\text { et al.,( }(2005)\end{array}$ & $\begin{array}{l}\text { Hsu et al., } \\
\text { (2005) }\end{array}$ & $\begin{array}{c}\text { Padoa- } \\
\text { Schioppa \& } \\
\text { Assad, (2006) }\end{array}$ & $\begin{array}{l}\text { McClure et } \\
\text { al., (2007) }\end{array}$ & $\begin{array}{l}\text { Knutson et } \\
\text { al., (2007) }\end{array}$ \\
\hline
\end{tabular}

Figura 1. Diagrama de árbol. 


\section{Resultados y discusión}

3.1 Revistas, autores y publicaciones más relevantes en neuroeconomía

En la Figura 2, se ilustra la producción científica asociada al tema de Neuroeconomía desde el año 2005 hasta el 31 de diciembre de 2019, en las bases de datos Wos y Scopus. El periodo de más alta producción, fue entre los años 2008 y 2013. Sin embargo, a partir del año 2013 el número de las publicaciones va en descenso, esto implica, que es un campo de conocimiento que ya ha madurado, y el interés de la comunidad científica en explorarlo ha decaído.

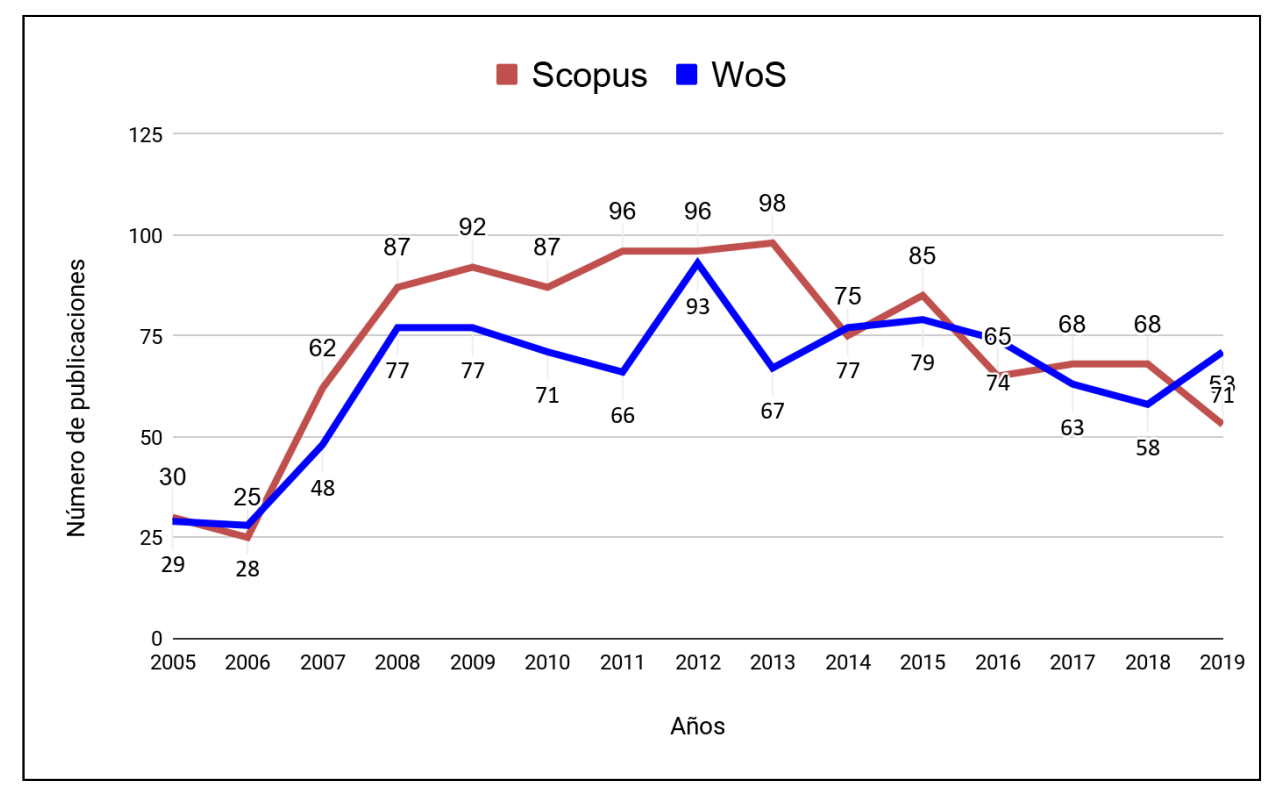

Figura 2. Producción científica anual en Neuroeconomía.

En relación al origen de las publicaciones, la figura 3 representa los diez países que lideran la producción académica global sobre Neuroeconomía. Es evidente el liderazgo y hegemonía de los Estados Unidos, ya que este país genera el $47 \%$ de las publicaciones del top 10 , mientras que los países Europeos reportan cerca del 20\% y los asiáticos el $5 \%$. 


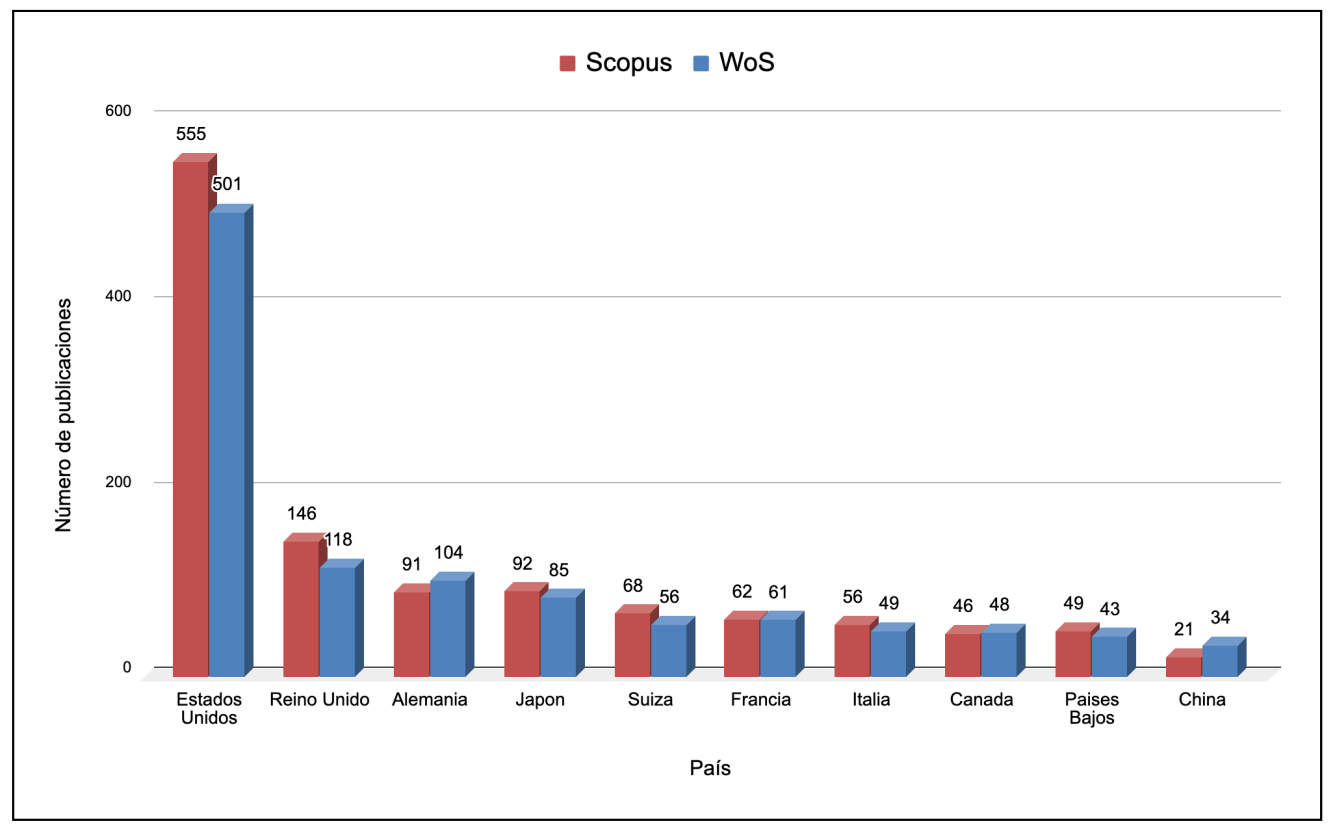

Figura 3. Los diez Países con mayor producción en neuroeconomía.

En la tabla 1, se relacionan las diez revistas científicas en las cuales se han publicado más artículos sobre Neuroeconomía, junto con el cuartil de cada una; nueve de ellas son revistas top, ya que están ubicadas en el cuartil Q1.

Tabla 1. Revistas más importantes en publicaciones sobre neuroeconomía.

\begin{tabular}{|c|c|c|c|}
\hline Revista & $\begin{array}{l}\text { Núm. } \\
\text { publicaciones }\end{array}$ & Cuartil & $\begin{array}{l}\text { Base de } \\
\text { datos }\end{array}$ \\
\hline \multirow[t]{2}{*}{ Frontiers in Neuroscience } & 31 & & Scopus \\
\hline & 35 & Q1 & Wos \\
\hline \multirow{2}{*}{$\begin{array}{l}\text { Proceedings of the National Academy of Sciences of } \\
\text { the United States of America }\end{array}$} & 34 & & Scopus \\
\hline & 34 & Q1 & WoS \\
\hline \multirow[t]{2}{*}{ Journal of Neuroscience } & 25 & & Scopus \\
\hline & 26 & Q1 & WoS \\
\hline \multirow[t]{3}{*}{ Neuroendocrinology Letters } & 19 & Q3 & Scopus \\
\hline & 19 & & WoS \\
\hline & 18 & & Scopus \\
\hline Economics and Philosophy & 23 & Q1 & WoS \\
\hline \multirow[t]{2}{*}{ Annals of the New York Academy of Sciences } & 18 & & Scopus \\
\hline & 18 & Q1 & WoS \\
\hline \multirow[t]{2}{*}{ Frontiers in Human Neuroscience } & 16 & & Scopus \\
\hline & 17 & Q1 & Wos \\
\hline \multirow[t]{2}{*}{ Neuroimage } & 15 & Q1 & Scopus \\
\hline & 17 & & WoS \\
\hline \multirow{3}{*}{$\begin{array}{l}\text { Philosophical Transactions of the Royal Society } \\
\text { Biological Sciences }\end{array}$} & 15 & Q1 & Scopus \\
\hline & 16 & & WoS \\
\hline & 13 & Q1 & Scopus \\
\hline Frontiers in Behavioral Neuroscience & 13 & & WoS \\
\hline
\end{tabular}


En la tabla 1 se identifica un considerable número de las publicaciones relacionadas a la Neuroeconomía, que se encuentran vinculadas en revistas del área de la salud. Según la categorización realizada por WoS, el $41 \%$ de los documentos hacen parte de la categoría de las neurociencias y la neurología, el $23 \%$ a la psicología, y tan solo el $24 \%$ al área de los negocios y la economía.

En la tabla 2 se relacionan los principales autores, de acuerdo con la cantidad de publicaciones y el índice $\mathrm{H}$. El autor con mayor cantidad de publicaciones es Taiki Takahashi, profesor de la
Universidad de Tokio, Japón, quien en Scopus y WoS tiene el número más alto de publicaciones. Sin embargo, Ernst Fehr, profesor en la Universidad de Zurich, cuyas líneas de investigación son: economía del comportamiento, neuroeconomía, economía experimental, psicología evolutiva y biología evolutiva; o Colin F Camerer, profesor del instituto de tecnología de california, investigador en economía, teoría de juego y neurociencia, son los que tienen un índice $\mathrm{H}$ más elevado. Es decir, aunque estos dos autores han publicado menos artículos, la calidad de sus documentos por cantidad de citas es mayor.

Tabla 2. Principales autores en neuroeconomía.

\begin{tabular}{lcclcc}
\hline \multicolumn{2}{c}{ Scopus } & & & WoS \\
\hline Autor & Núm. publicaciones & H-index & Autor & Núm. publicaciones & H-index \\
Takahashi, T. & 57 & 22 & Takahashi, T. & 51 & 13 \\
Camerer, C.F. & 22 & 76 & Camerer, C.F. & 17 & 66 \\
Glimcher, P.W. & 18 & 45 & Tobler, P.N. & 16 & 31 \\
Huettel, S.A. & 17 & 50 & Huettel, S.A. & 14 & 48 \\
Tobler, P.N. & 16 & 32 & Montague, P.R. & 14 & 53 \\
Fehr, E. & 15 & 80 & Rangel, A. & 14 & 48 \\
Platt, M.L. & 14 & 49 & Hsu, M. & 13 & 14 \\
Rustichini, A. & 14 & 40 & Kenning, P. & 13 & 23 \\
Sanfey, A.G. & 14 & 36 & Plassmann, H. & 12 & 18 \\
McCabe, K. & 13 & 9 & Rustichini, A. & 12 & 37 \\
\hline
\end{tabular}

En la figura 4, se identifican dos clústeres o grupos de co-citaciones. El primero y más grande, está integrado por: Kahneman, Knutson, Mcclure, Glimcher, Schultz y Bechara, entre otros, siendo el primero de la lista el autor más citado dentro de la red, y que a su vez es el más alto dentro de los autores relevantes en el campo. El segundo grupo está compuesto por Sanfey, Camerer, Fehr y Rilling. 


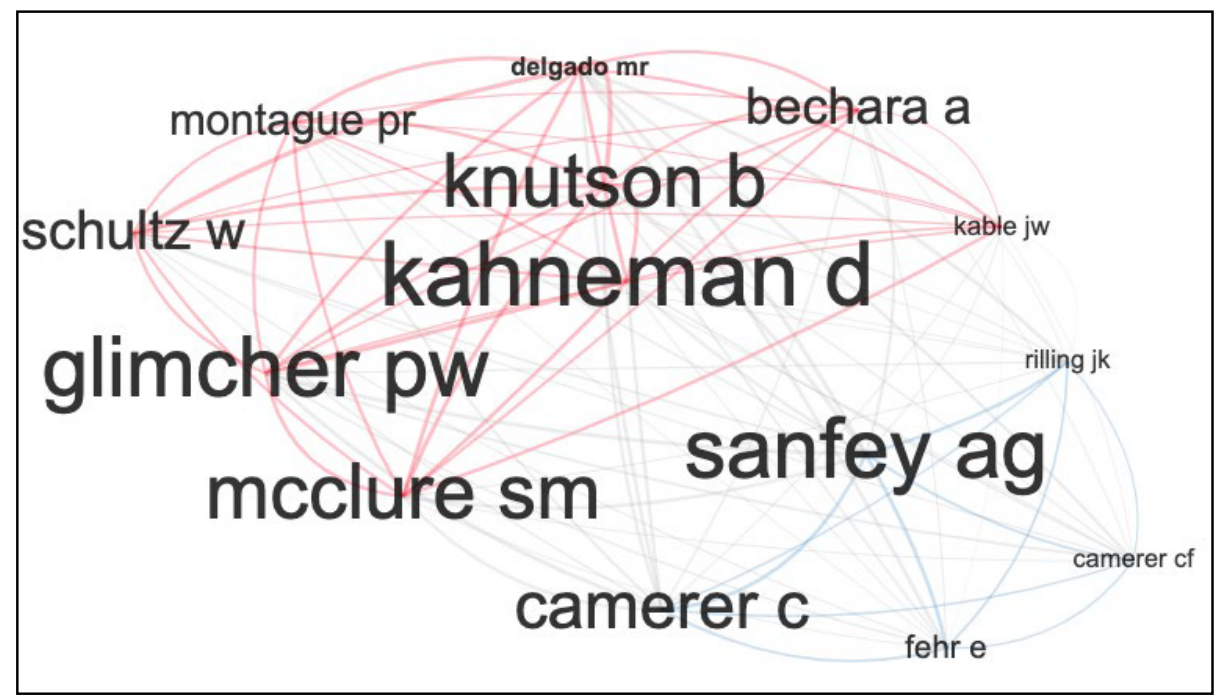

Figura 4. Red de co-citaciones.

La red co-citaciones de la Figura 5, muestra 5 grupos (enfoques), de la neuroeconomía. La red tiene 4349 nodos (artículos), y 14903 enlaces (referencias entre artículos). Los grupos conforman el $70 \%$ de la red, considerándo pertinente seleccionarlos como perspectivas. Los documentos con mayor citación, de acuerdo con la red, se pueden clasificar en dos grupos: en el primero se encuentran autores que han realizado apor- tes teóricos considerables a la neuroeconomía (Kahneman \& Tversky, 1979); mientras que el segundo está integrado por investigaciones empíricas sobre neuroeconomía, enmarcadas en aplicaciones bajo escenarios controlados, encontrándose múltiples trabajos, entre los que se destacan por citación: Camerer et al. (2005), McClure et al. (2004), y Sanfey et al. (2003).

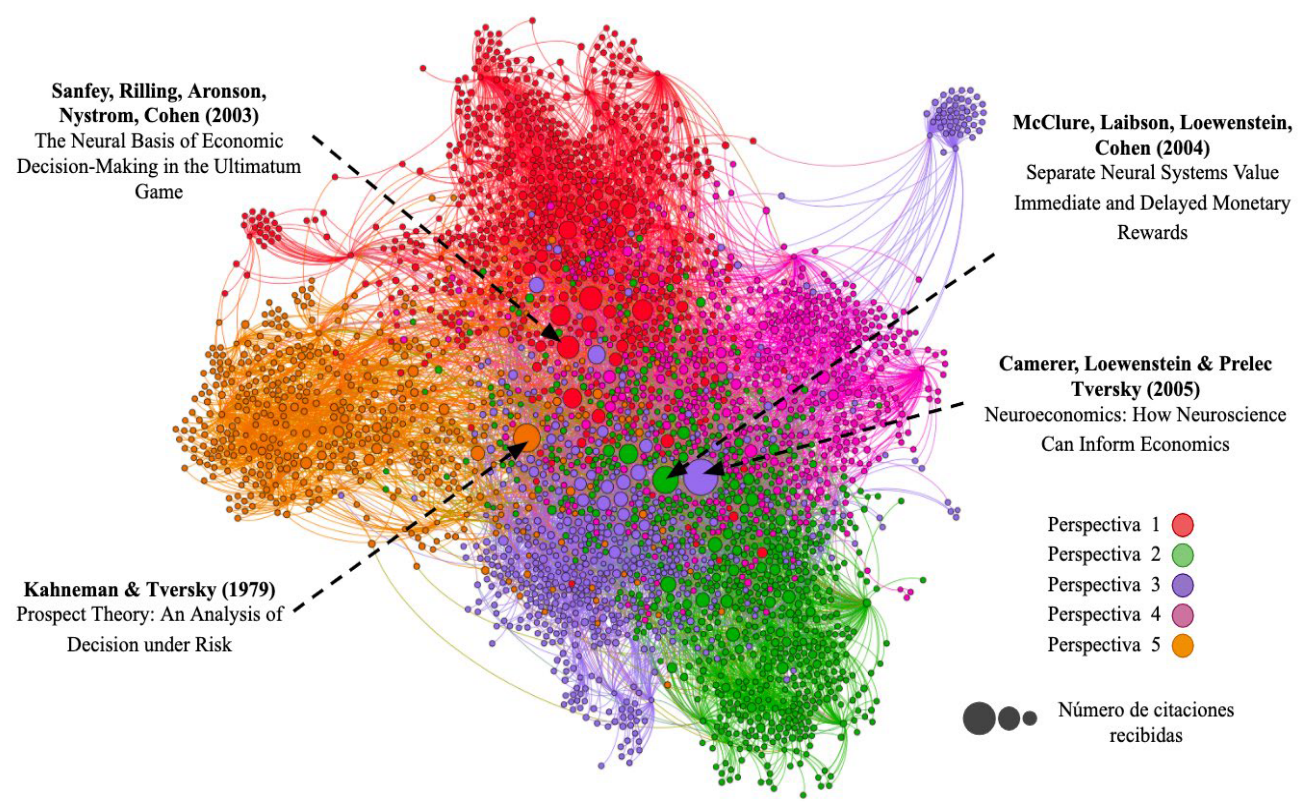

Figura 5. Red Neuroeconomía. 
A continuación, se presenta una selección de artículos considerados como los principales documentos, teniendo en cuenta los análisis previos y que se encuentran como documentos seminales (Ver tabla 1).

De los documentos que componen la raíz, el de mayor impacto en el tema de neuroeconomía desde el campo teórico, es: "Prospect Theory: an Analysis of decision under risk". Este artículo, publicado por Kahneman y Tversky (1979), desarrolla una crítica de la teoría de la utilidad esperada, y presenta un modelo teórico de la perspectiva de las decisiones bajo riesgo. Además, los autores describieron el "efecto certeza" y cómo la aversión al riesgo se involucra con la utilidad esperada. De otro lado, Sanfey et al. (2003), utilizaron la resonancia magnética funcional en jugadores del juego ultimatum, buscando identificar los sustratos neuronales de los procesos cognitivos y emocionales involucrados en la toma de decisiones económicas, analizando a los jugadores mientras responden propuestas justas e injustas.

Otras publicaciones, como las de Knutson et al. (2007), y McClure (2004), realizaron resonancias magnéticas a sujetos bajo escenarios controlados, analizando cómo la recompensa afecta los valores de las elecciones en toma de decisiones, para finalmente identificar el proceso relacionado con la activación de circuitos neuronales, y asociar de manera anticipada las decisiones de consumo. Asimismo, Glimcher y Rustichini (2004), realizaron una revisión y establecieron un marco teórico, en el que se involucran: la economía, psicología y neurociencia, con el proceso de decisión, buscando unificar teorías del comportamiento humano, proponiendo que la neuroeconomía se encarga de proporcionar teorías para estudiar la elección humana.

Finalmente, los castigos, las recompensas, los juegos de confianza, sensibilidad a las ganancias y las pérdidas, el análisis de la información y la teoría de la elección, fueron los tópicos de investigación para autores, como: Camerer et al. (2005), Hsu et al. (2005), McClure et al. (2007), Padoa-Schioppa y Assad (2006), y Schultz et al. (1997). Gracias a los documentos que hacen parte del tronco (Estructurales), del árbol de la neuroeconomía, se identificó una tendencia a desarrollar investigaciones de corte teórico, cuyo interés radicó en la revisión y proposición de modelos metodológicos neuroeconómicos, en los que se explican elementos de la economía tradicional, como el comportamiento de las elecciones (Camerer, 2013; Fehr \& Rangel, 2011; Fumagalli, 2016; Kenning \& Plassmann, 2005; Loewenstein et al., 2008; Monterosso et al., 2012; Sanfey, 2007). También se identificaron documentos con investigaciones empíricas, en las que se analizaron la toma de decisiones, mediante relaciones de: percepción de riesgo, producción hormonal, consumo de energía y uso de nuevas tecnologías en la neurociencia aplicada (Delgado \& Dickerson, 2012; Kishida et al., 2010; Kopton \& Kenning, 2014).

En los documentos que corresponden a las hojas, es clara la concentración de investigaciones cuyo objetivo es el desarrollo de metodologías y análisis de modelos conceptuales, en: la toma de decisiones, deficiencia en la toma de decisiones y la utilidad esperada. En ellos se encuentran relaciones de la psicología y la neuroeconomía, con el estudio de los componentes emocionales en la toma de decisiones, considerando el impacto de los fármacos y las estimulaciones eléctricas (Delgado et al., 2008; Fumagalli, 2017; Lin \& Vartanian, 2018; Mellick et al., 2015; Scherbaum et al., 2008; Shizgal, 2012; Sonuga-Barke et al., 2016; Westbrook \& Braver, 2015; Wilson et al., 2015; Yu \& Zhou, 2007).

\subsection{Perspectivas}

Mediante el proceso de clusterización, se identificaron 5 líneas principales de investigación (perspectivas), que representan el $70,2 \%$ de los artículos de la red (ver tabla 3). A continuación se describen las perspectivas identificadas. 
Tabla 3. Relación entre perspectivas y la red.

\section{Perspectiva}

Elecciones Económicas

707 nodos

1851 aristas

$16 \%$ red

Elección Social

697 nodos

1617 aristas

$16 \%$ red

Consideraciones sobre la neuroeconomía

572 nodos

1280 aristas

$13,2 \%$ red

Neurociencia del

consumidor

545 nodos

1066 aristas

$12,5 \%$ red

Comportamiento y

estímulo cerebral

545 nodos

1066 aristas

$12,5 \%$ red
Red
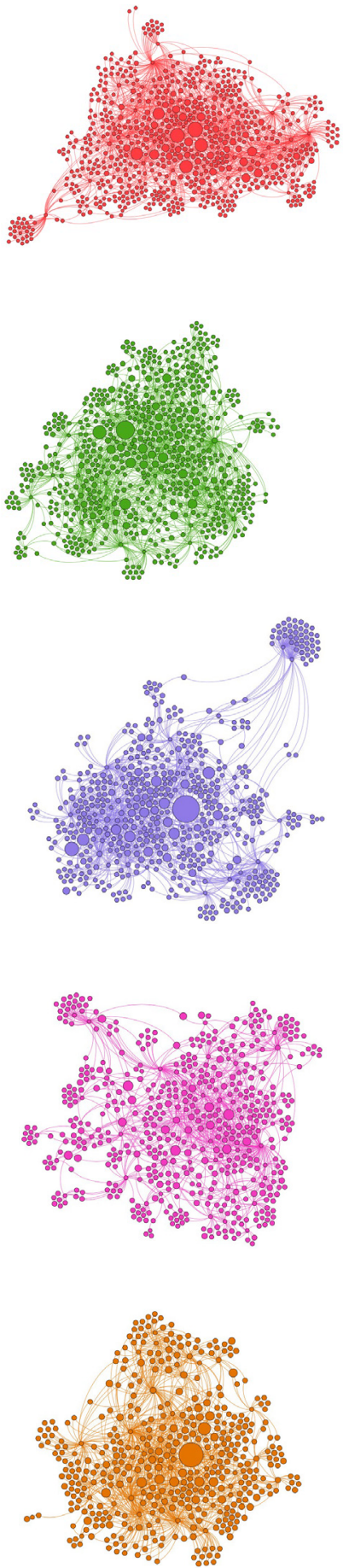

Nube de palabras
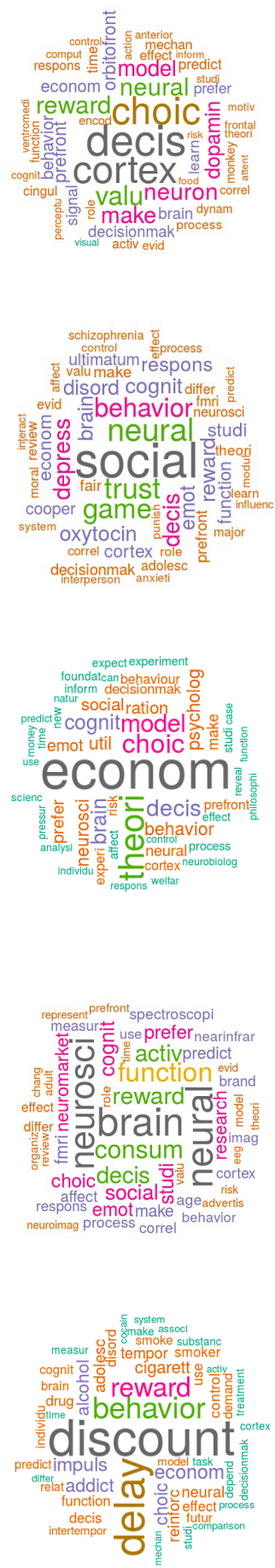


\section{Elecciones económicas}

Se propone definir el concepto de neuroeconomía y explicar, desde la biología, el comportamiento humano en la toma de decisiones (Rangel et al., 2008), quienes también proponen el uso de un léxico común. Los autores hegemónicos presentan estudios en los que se analizan las elecciones económicas y las preferencias subjetivas, en las que se asigna valor a las alternativas. Las áreas del cerebro más involucradas en este tipo de actividades, son la corteza prefrontal y la corteza orbitofrontal (Bartra et al., 2013; Levy \& Glimcher, 2012). Otros autores hablan sobre la importancia del riesgo en la toma de decisiones, las hormonas y los procesos de elección irracional (Beeler \& Mourra, 2018; Louie et al., 2013; Pearson et al., 2014; Raghuraman \& Padoa-Schioppa, 2014).

De otro lado, Krajbich y Dean (2015), afirman que la influencia de la neuroeconomía en la economía convencional ha sido poca, ya que la mayoría de los economistas no se encuentran interesados en el proceso de toma de decisiones, y que la influencia de la neurociencia en la economía será de corto plazo. Posteriormente, Konovalov y Krajbich (2019), autores estructurales de la perspectiva, manifiestan que la revolución de la neuroeconomía es una promesa fallida, dado el nivel de crecimiento desde el año 2005.

\section{Elección social}

La segunda perspectiva se caracteriza por estudiar la neuroeconomía y la relación con: la elección social, la psicología y la psiquiatría. En la raíz de la perspectiva, se encuentra el uso de la teoría de juegos en la toma de decisiones sociales, reconociendo la neuroeconomía como herramienta para analizar los procesos cerebrales, cuyo objetivo final es proporcionar una teoría única y general del comportamiento humano (Rilling \& Sanfey, 2011; Sharp et al., 2012). El estudio de las áreas cerebrales relacionadas con: las emociones, la percepción de riesgo, los comportamientos de negociación y la confianza, complementan las investigaciones del grupo (Kosfeld et al., 2005;
Mohr et al., 2010). En el tronco se identifican investigaciones teóricas, en las que se analiza: la racionalidad, la confianza, las interacciones sociales y los componentes hormonales que intervienen en la toma de decisiones, al igual que investigaciones que estudian el cerebro, identificando la brecha entre la depresión y la recompensa social (Mellick et al., 2015; Scheele et al., 2013). Lo anterior, sin dejar de lado el estudio de cómo las relaciones sociales afectan la actividad funcional y la conexión con otras áreas del cerebro, resaltando que la socialidad facilita la cooperación, la equidad y la provisión (Lee \& Harris, 2013; Lukinova \& Myagkov, 2016).

En las hojas se encuentran documentos sobre las tensiones opuestas entre la razón y la emoción en la toma de decisiones, al igual que la contribución de la neuroeconomía, en: la interpretación del procesamiento, la evaluación de recompensas y estudio de casos psiquiátricos (Robson et al., 2020). También se encuentra la contribución de la neurociencia social, a: la economía, la salud y el derecho, tres dominios con clara relevancia social (Kedia et al., 2017); hasta el punto de realizar la propuesta de un estudio neurosocioeconómico, que contribuya al análisis de los resultados de estas tres ramas del conocimiento (Krueger \& Meyer-Lindenberg, 2019), llegando al punto de realizar pruebas en personas con trastornos psicológicos y psiquiátricos (Sonuga-Barke et al., 2016).

\section{Consideraciones sobre la neuroeconomía}

La tercer perspectiva, permite reconocer en la nube de palabras el enfoque correspondiente a la aplicación directa sobre la economía. Los documentos hegemónicos, infieren que la economía está cada vez más influenciada por un enfoque de sistemas múltiples, para: la toma de decisiones, juicios, bases neuronales de la previsibilidad en perspectivas conductuales, el valor, la incertidumbre y la utilidad esperada. Existe una perspectiva fuertemente arraigada entre la psicología y la neurociencia, que contribuye a la comprensión del comportamiento (Camerer, 2007; Loewenstein et al., 2008; Padoa-Schioppa, 2011). 
En contraste, Harrison (2008), propone una reconsideración crítica a la neuroeconomía, planteando que es una exageración académica del marketing y, que sin distracciones, todavía existe potencial por descubrir. La neuroeconomía es un campo valioso de investigación, pero no en la forma en que se está desarrollando y vendiendo hoy. Los documentos estructurales, se caracterizan por: presentar, aplicar y discutir modelos de decisión y elección aplicados en neuroeconomía, en los que las restricciones metodológicas afectan los resultados (Fumagalli, 2014; Volk \& Köhler, 2012). Otros, como Dietrich y List (2016), y Okasha (2016), defienden el mentalismo como una visión para capturar fenómenos reales, a la par de los no observables en la ciencia (como electrones y campos electromagnéticos), y proponen esta ciencia positiva, como la que mejor se ajusta a la práctica científica. Estos autores rechazan que el comportamiento debe explicarse en términos de procesos cerebrales, a diferencia de la visión neuroeconómica radical.

Los documentos recientes, realizan una crítica descriptiva y normativa de la reconstrucción de la relación entre economía y neuropsicología, además de un recuento histórico, desde el nacimiento hasta los desarrollos recientes del campo de la economía experimental. Asimismo, evalúan los alcances de la investigación interdisciplinaria de la teoría económica y la neurobiología, comparando la neuroeconomía con la economía experimental (Rakviashvili, 2015; Serra, 2012). McMaster y Novarese (2016), realizan una crítica ante la posibilidad de crear una teoría conductual unificada, recurriendo a la neurociencia, psicología y la teoría económica. Estos autores argumentan que la neuroeconomía es sensible a problemas de subdeterminación, por lo tanto, parece interpretar en exceso los resultados experimentales.

\section{Neurociencia del consumidor}

En la cuarta perspectiva, se identifica el estudio de la incertidumbre, las recompensas y las preferencias en el estudio del cerebro. McClure et al. (2004), infieren que los seres humanos muestran preferencias subjetivas por una persona u otra. Es decir, los mensajes culturales se combinan con el contenido para dar forma a las percepciones, e incluso modificar el comportamiento para una recompensa primaria. Los cálculos heurísticos utilizados para emitir juicios bajo incertidumbre, como: representatividad, disponibilidad de instancias o escenarios, y ajuste de ancla, aunque son altamente económicos, conducen a errores sistemáticos y predecibles. En este sentido, varios autores han indicado que las activaciones neuronales se correlacionan con la predicción del riesgo (Poldrack, 2006; Preuschoff et al., 2008; Tversky \& Kahneman, 1974). En el tronco de la perspectiva, estos autores proponen que el énfasis actual de la investigación debe extenderse de la teoría a la práctica del marketing.

En adición, se plantea que los métodos científicos pueden aplicarse de manera eficiente al marketing. De esta manera, la neurociencia se convierte en un complemento importante para la investigación del consumidor. La espectroscopía de infrarrojo cercano funcional (FNIRS, por sus siglas en inglés), es una herramienta prometedora para la medición de los procesos de toma de decisiones económicas y la atención del consumidor (Holper et al., 2014; Kopton \& Kenning, 2014; Meyerding \& Risius, 2018).

Los datos neurofisiológicos deberían incluirse en la investigación realizada en los campos empresariales. La teoría neurocientífica, puede agregarse a las líneas de investigación existentes en marketing. Esto debido a que los datos del cerebro, pueden permitir la predicción el comportamiento a nivel de mercado, en una gran variedad de tipos de decisiones (Karmarkar \& Plassmann, 2019; Levallois et al., 2019). Las técnicas neurológicas y fisiológicas, incluyen: resonancia magnética funcional, magnetoencefalografía, electroencefalografía, seguimiento ocular, respuesta galvánica de la piel, frecuencia cardíaca, reconocimiento facial y prueba de asociación implícita, con la finalidad de obtener información para estudiar los procesos de análisis y decisión (Harris et al., 2018; Ramsoy et al., 2017). 


\section{Comportamiento y estímulo cerebral}

La última perspectiva, en sus raíces, habla sobre el estudio del consumo de sustancias y los sistemas cerebrales. En las elecciones de recompensa monetaria, se involucran dos sistemas separados: la parte del sistema límbico, relacionado con el sistema de dopamina incluida la corteza paralímbica, se activa en decisiones de recompensa de inmediato; la región de la corteza prefrontal lateral y corteza parietal posterior, están asociadas a elecciones intertemporales, independiente de la demora (McClure et al., 2004). La adicción al consumo de drogas, es el producto del desequilibrio entre dos sistemas neuronales separados pero interactivos. Un sistema impulsivo de amígdala para indicar el placer o dolor inmediato, y un sistema de corteza prefrontal reflexivo para indicar el placer o dolor a futuro (Bickel, et al., 2007; Kangas \& Walker, 2008).

Las investigaciones indican que los sistemas cerebrales admiten recompensas demoradas. Los individuos con trastornos por consumo de alcohol, muestran mayor hiperactividad en regiones asociadas al control cognitivo (corteza prefrontal dorsolateral), y pensamiento prospectivo (Amlung et al., 2014; MacKillop et al., 2014). Las diferencias individuales en la toma de decisiones, están relacionadas con aspectos de neuroanatomía y neurofisiología. En este sentido, las características neurobiológicas podrían ser adecuadas como biomarcadores para los rasgos de decisión. Además, los marcadores neuronales podrían ayudar a predecir el comportamiento económico (Kable \& Levy, 2015).

De otro lado, el consumo de sustancias adictivas promueve el egoísmo y hace más propensos a los consumidores a elegir recompensas más pequeñas e inmediatas, en lugar de recompensas demoradas más grandes (Lawn et al., 2019; Wilson et al., 2015). Alonso (2013), Menciona que las decisiones intertemporales involucran números, debido a que los sujetos deciden entre recompensas, con diferentes demoras y probabilidades. Nacen dos postulados: i) las decisiones intertemporales no son sobre el tiempo sino sobre comparaciones de números, y ii) las decisiones intertemporales son sobre el tiempo, pero con números relevantes comprimidos en su forma logarítmica.

\section{Conclusiones}

El interés por parte de la comunidad científica en torno a temas de investigación en neuroeconomía, es evidente. En los últimos años ha venido teniendo un alto crecimiento, reportando una tasa anual del 25\%, reconociéndose el año 2012 como el año con mayor producción científica en el tema. Daniel Kahneman y Amos Tversky, son los autores con mayor número de referencias, por lo que puede decirse que son los autores más reconocidos. Por otro lado, Takahashi, es uno de los autores que más producción tiene en el tema. En cuanto a los países, los de mayor productividad son Estados Unidos y el Reino Unido. Se evidencia la importancia del riesgo en la toma de decisiones y cómo interviene en los procesos de elección. También cómo se determinan las preferencias de consumo y las áreas del cerebro involucradas en la toma de decisiones.

Analizar la racionalidad, la confianza, las interacciones sociales y hasta los componentes hormonales que intervienen en la toma de decisiones, ha permitido reconocer la brecha entre la depresión y la recompensa social. No se pueden dejar de lado los estudios de cómo las relaciones sociales afectan las conexiones cerebrales, ya que las relaciones sociales, fomentan: la cooperación, la equidad y la provisión.

Es pertinente presentar, aplicar y discutir modelos de decisión y elección aplicados a la neuroeconomía, ya que las restricciones metodológicas afectan los resultados y, en la actualidad, no se ha llegado a un consenso metodológico. La neuroeconomía ha tenido una extensión sobre el marketing, llamándose neuromarketing; son propuestas de uso eficiente de esta área del conocimiento, y se convierte en un complemento importante para la investigación del consumidor. El uso de FNIRS, es una herramienta prometedora en la medición de toma de decisiones y la atención del consumidor. 
Los sistemas cerebrales admiten recompensas demoradas. Los individuos con trastornos por consumo de alcohol muestran mayor hiperactividad en regiones asociadas al control cognitivo (corteza prefrontal dorsolateral), y pensamiento prospectivo. Las diferencias individuales en la toma de decisiones están relacionadas con aspectos de neuroanatomía y neurofisiología. Las características neurobiológicas podrían ser adecuadas como biomarcadores para los rasgos de decisión. Los marcadores neuronales podrían ayudar a predecir el comportamiento económico.

Como futuras líneas de investigación, se reconocen tendencias sobre investigaciones aplicadas al marketing, la elección social, la toma de decisiones relacionada con economía, consumo de sustancias psicoactivas y estados mentales. Analizando los resultados de Rakviashvili (2015), Westbrook y Braver (2015), y Fumagalli (2014), Es necesario estandarizar y encontrar una metodología de investigación en la que converjan los criterios para fortalecer los resultados de las investigaciones realizadas, ya que no se pueden desconocer las limitaciones de las metodologías actuales.

\section{Referencias}

Addor, N., \& Melsen, L. A. (2019). Legacy, Rather Than Adequacy, Drives the Selection of Hydrological Models. Water Resources Research, 55 (1), 378-390. https://doi.org/10.1029/2018WR022958

Almeida, F., \& de Paula, L. G. (2019). The Place of Uncertainty in Heterodox Economics Journals: A Bibliometric Study. Journal of Economic Issues, 53 (2), 553-562. https://doi.org/10.1080/0021362 4.2019 .1603771

Alonso, S. (2013). Temporal Discounting and Number Representation. Journal of Behavioral Finance, 14 (3), 240-251. https://doi.org/10.1080 $/ 15427560.2013 .820188$

Amlung, M., Sweet, L. H., Acker, J., Brown, C. L., \& Mackillop, J. (2014). Dissociable brain signatures of choice conflict and immediate reward preferences in alcohol use disorders. Addiction Biology, 19 (4), 743-753. https://doi.org/10.1111/ adb.12017

Aria, M., \& Cuccurullo, C. (2017). bibliometrix : An R-tool for comprehensive science mapping analysis. Journal of Informetrics, 11 (4), 959-975. https://doi.org/10.1016/j.joi.2017.08.007

Bartra, O., McGuire, J. T., \& Kable, J. W. (2013). The valuation system: a coordinate-based metaanalysis of BOLD fMRI experiments examining neural correlates of subjective value. Neurolmage, 76, 412-427. https://doi.org/10.1016/j. neuroimage.2013.02.063

Beeler, J. A., \& Mourra, D. (2018). To Do or Not to Do: Dopamine, Affordability and the Economics of Opportunity. Frontiers in Integrative Neuroscience, 12, 6. https://doi.org/10.3389/ fnint.2018.00006

Bickel, W. K., Miller, M. L., Yi, R., Kowal, B. P., Lindquist, D. M., \& Pitcock, J. A. (2007). Behavioral and neuroeconomics of drug addiction: competing neural systems and temporal discounting processes. Drug and Alcohol Dependence, 90 Suppl 1, S85-S91. https://doi.org/10.1016/j.drugalcdep.2006.09.016

Blondel, V. D., Guillaume, J.-L., Lambiotte, R., \& Lefebvre, E. (2008). Fast unfolding of communities in large networks. Journal of Statistical Mechanics: Theory and Experiment, 2008 (10), P10008. https:// doi.org/10.1088/1742-5468/2008/10/p10008

Bond, M., \& Buntins, K. (2018). An analysis of the Australasian Journal of Educational Technology 2013-2017. Australasian Journal of Educational Technology. https://doi.org/10.14742/ajet.4359

Buitrago, S., Duque, P., \& Robledo, S. (2020). Branding Corporativo: una revisión bibliográfica. ECONÓMICAS CUC, 41(1). https://doi.org/10.17981/ econcuc.41.1.2020.0rg.1

Camerer, C. F. (2007). Neuroeconomics: Using Neuroscience to Make Economic Predictions. The Economic Journal of Nepal, 117(519), C26-C42. https://doi.org/10.1111/j.1468-0297.2007.02033.x 
Camerer, C. F. (2013). A Review Essay about Foundations of Neuroeconomic Analysis by Paul Glimcher. Journal of Economic Literature, 51(4), 1155-1182. https://doi.org/10.1257/jel.51.4.1155

Camerer, C., Loewenstein, G., \& Prelec, D. (2005). Neuroeconomics: How Neuroscience Can Inform Economics. Journal of Economic Literature, 43(1), 9-64. https://doi.org/10.1257/0022051053737843

D’Angiulli, A., Lipina, S. J., \& Olesinska, A. (2012). Explicit and implicit issues in the developmental cognitive neuroscience of social inequality. Frontiers in Human Neuroscience, 6, 254. https:// doi.org/10.3389/fnhum.2012.00254

Delgado, M. R., \& Dickerson, K. C. (2012). Rewardrelated learning via multiple memory systems. Biological Psychiatry, 72(2), 134-141. https://doi. org/10.1016/j.biopsych.2012.01.023

Delgado, M. R., Li, J., Schiller, D., \& Phelps, E. A. (2008). The role of the striatum in aversive learning and aversive prediction errors. Philosophical Transactions of the Royal Society of London. Series B, Biological Sciences, 363(1511), 3787-3800. https://doi.org/10.1098/rstb.2008.0161

Dietrich, F., \& List, C. (2016). MENTALISM VERSUS BEHAVIOURISM IN ECONOMICS: A PHILOSOPHYOF-SCIENCE PERSPECTIVE. Economics and Philosophy, 32(2), 249-281. https://doi.org/10.1017/ S0266267115000462

Duque, P., \& Cervantes-Cervantes, L.-S. (2019). Responsabilidad Social Universitaria: una revisión sistemática y análisis bibliométrico. Estudios Gerenciales, 451-464. https://doi.org/10.18046/j. estger.2019.153.3389

Fehr, E., \& Rangel, A. (2011). Neuroeconomic Foundations of Economic Choice-Recent Advances. The Journal of Economic Perspectives: $A$ Journal of the American Economic Association, 25(4), 3-30. https://doi.org/10.1257/jep.25.4.3

Fumagalli, R. (2014). Neural Findings and Economic Models: Why Brains Have Limited Relevance for Economics. Philosophy of the Social Sciences, 44(5), 606-629. https://doi. org/10.1177/0048393114530948
Fumagalli, R. (2016). Five theses on neuroeconomics. Journal of Economic Methodology, 23(1), 77-96. https://doi.org/10.1080/1350178X.2015. 1024883

Fumagalli, R. (2017). On the neural enrichment of economic models: recasting the challenge. Biology \& Philosophy, 32(2), 201-220. https://doi. org/10.1007/s10539-016-9546-y

Glimcher, P. W., \& Rustichini, A. (2004). Neuroeconomics: the consilience of brain and decision. Science, 306(5695), 447-452. https://doi. org/10.1126/science.1102566

Harris, J. M., Ciorciari, J., \& Gountas, J. (2018). Public health social media communications and consumer neuroscience. Cogent Psychology, 5(1), 888. https://doi.org/10.1080/23311908.2018.14 34058

Harrison, G. W. (2008). NEUROECONOMICS: A CRITICAL RECONSIDERATION. Economics and Philosophy, 24(3), 303-344. https://doi.org/10.1017/ S0266267108002009

Holper, L., Wolf, M., \& Tobler, P. N. (2014). Comparison of functional near-infrared spectroscopy and electrodermal activity in assessing objective versus subjective risk during risky financial decisions. Neurolmage, 84, 833-842. https://doi. org/10.1016/j.neuroimage.2013.09.047

Hsu, M., Bhatt, M., Adolphs, R., Tranel, D., \& Camerer, C. F. (2005). Neural systems responding to degrees of uncertainty in human decisionmaking (Review of Neural systems responding to degrees of uncertainty in human decisionmaking). Science, 310(5754), 1680-1683. https:// doi.org/10.1126/science.1115327

Kable, J. W., \& Levy, I. (2015). Neural markers of individual differences in decision-making. Current Opinion in Behavioral Sciences, 5, 100-107. https:// doi.org/10.1016/j.cobeha.2015.08.004

Kahneman, D., \& Tversky, A. (1979). Prospect Theory: An Analysis of Decision under Risk. Econometrica: Journal of the Econometric Society, 47(2), 263. https://doi.org/10.2307/1914185 
Kangas, B. D., \& Walker, D. J. (2008). An adjustingdose procedure for assessing the reinforcing effects of nitrous oxide with humans. Pharmacology, Biochemistry, and Behavior, 91(1), 104-108. https://doi.org/10.1016/j.pbb.2008.06.017

Karmarkar, U. R., \& Plassmann, H. (2019). Consumer Neuroscience: Past, Present, and Future. Organizational Research Methods, 22(1), 174-195. https://doi.org/10.1177/1094428117730598

Kedia, G., Harris, L., Lelieveld, G.-J., \& van Dillen, L. (2017). From the Brain to the Field: The Applications of Social Neuroscience to Economics, Health and Law. Brain Sciences, 7(8). https://doi. org/10.3390/brainsci7080094

Kenning, P., \& Plassmann, H. (2005). Neuroeconomics: an overview from an economic perspective. Brain Research Bulletin, 67(5), 343-354. https:// doi.org/10.1016/j.brainresbull.2005.07.006

Kishida, K. T., King-Casas, B., \& Montague, P. R. (2010). Neuroeconomic approaches to mental disorders. Neuron, 67(4), 543-554. https://doi. org/10.1016/j.neuron.2010.07.021

Knutson, B., Rick, S., Wimmer, G. E., Prelec, D., \& Loewenstein, G. (2007). Neural predictors of purchases. Neuron, 53(1), 147-156. https://doi. org/10.1016/j.neuron.2006.11.010

Konovalov, A., \& Krajbich, I. (2019). Over a Decade of Neuroeconomics: What Have We Learned? Organizational Research Methods, 22(1), 148-173. https://doi.org/10.1177/1094428116644502

Kopton, I. M., \& Kenning, P. (2014). Near-infrared spectroscopy (NIRS) as a new tool for neuroeconomic research. Frontiers in Human Neuroscience, 8, 549. https://doi.org/10.3389/ fnhum.2014.00549

Kosfeld, M., Heinrichs, M., Zak, P. J., Fischbacher, U., \& Fehr, E. (2005). Oxytocin increases trust in humans. Nature, 435(7042), 673-676. https://doi. org/10.1038/nature03701

Krajbich, I., \& Dean, M. (2015). How can neuroscience inform economics? Current Opinion in Behavioral Sciences, 5, 51-57. https://doi. org/10.1016/j.cobeha.2015.07.005
Krause, F., \& Lindemann, O. (2014). Expyriment: a Python library for cognitive and neuroscientific experiments. Behavior Research Methods, 46(2), 416-428. https://doi.org/10.3758/s13428-0130390-6

Krueger, F., \& Meyer-Lindenberg, A. (2019). Toward a Model of Interpersonal Trust Drawn from Neuroscience, Psychology, and Economics. Trends in Neurosciences, 42(2), 92-101. https://doi. org/10.1016/j.tins.2018.10.004

Landínez-Martínez, D. A., Robledo, S., \& Montoya-Londoño, D. M. (2019). Executive function performance in patients with obesity: a systematic review. Psychologia; an International Journal of Psychology in the Orient, 13(2), 121-134. https:// doi.org/10.21500/19002386.4230

Lawn, W., Mithchener, L., Freeman, T. P., Benattayallah, A., Bisby, J. A., Wall, M. B., Dodds, C. M., Curran, H. V., \& Morgan, C. J. A. (2019). Value-based decision-making of cigarette and nondrug rewards in dependent and occasional cigarette smokers: An FMRI study. Addiction Biology, e12802. https://doi.org/10.1111/adb.12802

Lee, V. K., \& Harris, L. T. (2013). How social cognition can inform social decision making. Frontiers in Neuroscience, 7, 259. https://doi.org/10.3389/ fnins.2013.00259

Levallois, C., Smidts, A., \& Wouters, P. (2019). The emergence of neuromarketing investigated through online public communications (20022008). Business History, 450, 1-40. https://doi.or $\mathrm{g} / 10.1080 / 00076791.2019 .1579194$

Levy, D. J., \& Glimcher, P. W. (2012). The root of all value: a neural common currency for choice. Current Opinion in Neurobiology, 22(6), 1027-1038. https://doi.org/10.1016/j.conb.2012.06.001

Lin, H., \& Vartanian, O. (2018). A Neuroeconomic Framework for Creative Cognition. Perspectives on Psychological Science: A Journal of the Association for Psychological Science, 13(6), 655-677. https://doi.org/10.1177/1745691618794945

Loewenstein, G., Rick, S., \& Cohen, J. D. (2008). Neuroeconomics. Annual Review of Psychology, 
59, 647-672. https://doi.org/10.1146/annurev. psych.59.103006.093710

Louie, K., Khaw, M. W., \& Glimcher, P. W. (2013). Normalization is a general neural mechanism for context-dependent decision making. Proceedings of the National Academy of Sciences of the United States of America, 110(15), 6139-6144. https://doi.org/10.1073/pnas.1217854110

Lukinova, E., \& Myagkov, M. (2016). Impact of Short Social Training on Prosocial Behaviors: An fMRI Study. Frontiers in Systems Neuroscience, 10, 60. https://doi.org/10.3389/fnsys.2016.00060

MacKillop, J., Amlung, M. T., Acker, J., Gray, J. C., Brown, C. L., Murphy, J. G., Ray, L. A., \& Sweet, L. H. (2014). The neuroeconomics of alcohol demand: an initial investigation of the neural correlates of alcohol cost-benefit decision making in heavy drinking men. Neuropsychopharmacology: Official Publication of the American College of Neuropsychopharmacology, 39(8), 1988-1995. https://doi.org/10.1038/npp.2014.47

Marín-López, J.C., Robledo, S., \& Duque-Méndez, N. D. (2017). Marketing Emprendedor: Una perspectiva cronológica utilizando Tree of Science. Revista Civilizar de Empresa Y Economía, 7(13), 113-123. https://revistas.usergioarboleda.edu. co/index.php/ceye/article/view/923

McClure, S. M., Ericson, K. M., Laibson, D. I., Loewenstein, G., \& Cohen, J. D. (2007). Time discounting for primary rewards. The Journal of Neuroscience: The Official Journal of the Society for Neuroscience, 27(21), 5796-5804. https://doi. org/10.1523/JNEUROSCI.4246-06.2007

McClure, S. M., Laibson, D. I., Loewenstein, G., \& Cohen, J. D. (2004). Separate neural systems value immediate and delayed monetary rewards. Science, 306(5695), 503-507. https://doi. org/10.1126/science. 1100907

McClure, S. M., Li, J., Tomlin, D., Cypert, K. S., Montague, L. M., \& Montague, P. R. (2004). Neural correlates of behavioral preference for culturally familiar drinks. Neuron, 44(2), 379-387. https:// doi.org/10.1016/j.neuron.2004.09.019
McKiernan, P. (2017). Prospective thinking; scenario planning meets neuroscience. Technological Forecasting and Social Change, 124, 66-76. https:// doi.org/10.1016/j.techfore.2016.10.069

McMaster, R., \& Novarese, M. (2016). Neuroeconomics: Infeasible and Underdetermined. Journal of Economic Issues, 50(4), 963-983. https://doi.org/1 0.1080/00213624.2016.1249745

Medina, J., Ojeda-Aciego, M., Verdegay, J. L., Perfilieva, I., Bouchon-Meunier, B., \& Yager, R. R. (Eds.). (2018). Information Processing and Management of Uncertainty in Knowledge-Based Systems. Applications: 17th International Conference, IPMU 2018, Cádiz, Spain, June 11-15, 2018, Proceedings, Part III (Vol. 855). Springer International Publishing. https://doi.org/10.1007/978-3-319-91479-4

Mellick, W., Sharp, C., \& Ernst, M. (2015). Neuroeconomics for the Study of Social Cognition in Adolescent Depression. Clinical Psychology: Science and Practice, 22(3), 255-276. https://doi. org/10.1111/cpsp.12106

Meyerding, S. G. H., \& Risius, A. (2018). Reading minds: Mobile functional near-infrared spectroscopy as a new neuroimaging method for economic and marketing research-A feasibility study. Journal of Neuroscience, Psychology, and Economics, 11(4), 197-212. https://doi.org/10.1037/ npe0000090

Mohr, P. N. C., Biele, G., \& Heekeren, H. R. (2010). Neural processing of risk. The Journal of Neuroscience: The Official Journal of the Society for Neuroscience, 30(19), 6613-6619. https://doi. org/10.1523/JNEUROSCI.0003-10.2010

Monterosso, J., Piray, P., \& Luo, S. (2012). Neuroeconomics and the study of addiction. Biological Psychiatry, 72(2), 107-112. https://doi. org/10.1016/j.biopsych.2012.03.012

Ohri, A. (2013). R for Business Analytics. Suiza: Springer Nature. https://doi.org/10.1007/978-14614-4343-8

Okasha, S. (2016). ON THE INTERPRETATION OF DECISION THEORY. Economics and Philo- 
sophy, 32(3), 409-433. https://doi.org/10.1017/ S0266267115000346

Padoa-Schioppa, C. (2011). Neurobiology of economic choice: a good-based model. Annual Review of Neuroscience, 34, 333-359. https://doi. org/10.1146/annurev-neuro-061010-113648

Padoa-Schioppa, C., \& Assad, J. A. (2006). Neurons in the orbitofrontal cortex encode economic value. Nature, 441(7090), 223-226. https://doi. org/10.1038/nature04676

Pearson, J. M., Watson, K. K., \& Platt, M. L. (2014). Decision making: the neuroethological turn. Neuron, 82(5), 950-965. https://doi.org/10.1016/j. neuron.2014.04.037

Poldrack, R. A. (2006). Can cognitive processes be inferred from neuroimaging data? Trends in Cognitive Sciences, 10(2), 59-63. https://doi. org/10.1016/j.tics.2005.12.004

Preuschoff, K., Quartz, S. R., \& Bossaerts, P. (2008). Human insula activation reflects risk prediction errors as well as risk. The Journal of Neuroscience: The Official Journal of the Society for Neuroscience, 28(11), 2745-2752. https://doi.org/10.1523/JNEUROSCI.4286-07.2008

Raghuraman, A. P., \& Padoa-Schioppa, C. (2014). Integration of multiple determinants in the neuronal computation of economic values. The Journal of Neuroscience: The Official Journal of the Society for Neuroscience, 34(35), 11583-11603. https://doi.org/10.1523/JNEUROSCl.1235-14.2014

Rakviashvili, A. (2015). Neurobiology and New Opportunities for Experimental Economics. Voprosy Ekonomiki, 12, 124-137. https://doi. org/10.32609/0042-8736-2015-12-124-137

Ramírez, M. H. (2010). Neurofinanzas: Cuando las decisiones financieras no son racionales. Tec Empresarial, 4(3), 21-30. Recuperado de: https:// dialnet.unirioja.es/servlet/articulo?codigo $=3398$ 008\&info=resumen\&idioma $=$ ENG

Ramsøy, T. Z., Jacobsen, C., Friis-Olivarius, M., Bagdziunaite, D., \& Skov, M. (2017). Predictive value of body posture and pupil dilation in assessing consumer preference and choice. Journal of Neuroscience, Psychology, and Economics, 10(2-3), 95-110. https://doi.org/10.1037/npe0000073

Rangel, A., Camerer, C., \& Montague, P. R. (2008). A framework for studying the neurobiology of value-based decision making. Nature Reviews. Neuroscience, 9(7), 545-556. https://doi. org/10.1038/nrn2357

Rilling, J. K., \& Sanfey, A. G. (2011). The neuroscience of social decision-making. Annual Review of Psychology, 62, 23-48. https://doi.org/10.1146/ annurev.psych.121208.131647

Robledo-Giraldo, S., Duque-Méndez, N. D., \& Zuluaga-Giraldo, J. I. (2013). Difusión de productos a través de redes sociales: una revisión bibliográfica utilizando la teoría de grafos. Respuestas, 18 (2), 28-42. https://doi.org/10.22463/0122820x.361

Robledo, S., Osorio, G., \& Lopez, C. (2014). Networking en pequeña empresa: una revisión bibliográfica utilizando la teoría de grafos. Investigación y Desarrollo, 11(2).

Robson, S. E., Repetto, L., Gountouna, V.-E., \& Nicodemus, K. K. (2020). A review of neuroeconomic gameplay in psychiatric disorders. Molecular Psychiatry, 25(1), 67-81. https://doi.org/10.1038/ s41380-019-0405-5

Sanfey, A. G. (2007). Social decision-making: insights from game theory and neuroscience. Science, 318(5850), 598-602. https://doi. org/10.1126/science.1142996

Sanfey, A. G., Rilling, J. K., Aronson, J. A., Nystrom, L. E., \& Cohen, J. D. (2003). The neural basis of economic decision-making in the Ultimatum Game. Science, 300(5626), 1755-1758. https://doi. org/10.1126/science.1082976

Scheele, D., Mihov, Y., Schwederski, O., Maier, W., \& Hurlemann, R. (2013). A negative emotional and economic judgment bias in major depression. European Archives of Psychiatry and Clinical Neuroscience, 263(8), 675-683. https://doi. org/10.1007/s00406-013-0392-5 
Scherbaum, S., Dshemuchadse, M., \& Kalis, A. (2008). Making decisions with a continuous mind. Cognitive, Affective \& Behavioral Neuroscience, 8(4), 454-474. https://doi.org/10.3758/CABN.8.4.454

Schultz, W., Dayan, P., \& Montague, P. R. (1997). A neural substrate of prediction and reward. Science, 275(5306), 1593-1599. https://doi.org/10.1126/ science. 275.5306 .1593

Serra, D. (2012). Un aperçu historique de l'économie expérimentale : des origines aux évolutions récentes. Revue D'économie Politique, 122(5), 749. https://doi.org/10.3917/redp.225.0749

Sharp, C., Monterosso, J., \& Montague, P. R. (2012). Neuroeconomics: a bridge for translational research. Biological Psychiatry, 72(2), 87-92. https:// doi.org/10.1016/j.biopsych.2012.02.029

Shizgal, P. (2012). Scarce means with alternative uses: robbins' definition of economics and its extension to the behavioral and neurobiological study of animal decision making. Frontiers in Neuroscience, 6, 20. https://doi.org/10.3389/ fnins.2012.00020

Smith, A. C., Frank, L. M., Wirth, S., Yanike, M., Hu, D., Kubota, Y., Graybiel, A. M., Suzuki, W. A., \& Brown, E. N. (2004). Dynamic analysis of learning in behavioral experiments. The Journal of Neuroscience: The Official Journal of the Society for Neuroscience, 24(2), 447-461. https://doi. org/10.1523/JNEUROSCI.2908-03.2004

Sonuga-Barke, E. J. S., Cortese, S., Fairchild, G., \& Stringaris, A. (2016). Annual Research Review: Transdiagnostic neuroscience of child and adolescent mental disorders--differentiating decision making in attention-deficit/hyperactivity disorder, conduct disorder, depression, and anxiety. Journal of Child Psychology and Psychiatry, and Allied Disciplines, 57(3), 321-349. https:// doi.org/10.1111/jcpp.12496

Trepel, C., Fox, C. R., \& Poldrack, R. A. (2005). Prospect theory on the brain? Toward a cognitive neuroscience of decision under risk. Brain Research. Cognitive Brain Research, 23(1), 34-50. https://doi.org/10.1016/j.cogbrainres.2005.01.016
Tversky, A., \& Kahneman, D. (1974). Judgment under Uncertainty: Heuristics and Biases. Science, 185(4157), 1124-1131. https://doi.org/10.1126/ science.185.4157.1124

Volk, S., \& Köhler, T. (2012). Brains and Games: Applying Neuroeconomics to Organizational Research. Organizational ResearchMethods, 15(4),522552. https://doi.org/10.1177/1094428112449656

Westbrook, A., \& Braver, T. S. (2015). Cognitive effort: A neuroeconomic approach. Cognitive, Affective \& Behavioral Neuroscience, 15(2), 395 415. https://doi.org/10.3758/s13415-015-0334-y

Wilson, A. G., Franck, C. T., Mueller, E. T., Landes, R. D., Kowal, B. P., Yi, R., \& Bickel, W. K. (2015). Predictors of delay discounting among smokers: education level and a Utility Measure of Cigarette Reinforcement Efficacy are better predictors than demographics, smoking characteristics, executive functioning, impulsivity, or time perception. Addictive Behaviors, 45, 124-133. https:// doi.org/10.1016/j.addbeh.2015.01.027

Yu, R., \& Zhou, X. (2007). Neuroeconomics: Opening the "black box" behind the economic behavior. Chinese Science Bulletin = Kexue Tongbao, 52(9), 1153-1161. https://doi.org/10.1007/s11434007-0193-1

Zuluaga, M., Robledo, S., Osorio-Zuluaga, G. A., Yathe, L., Gonzalez, D., \& Taborda, G. (2016). Metabolómica y Pesticidas: Revisión sistemática de literatura usando teoría de grafos para el análisis de referencias. Nova, 14(25), 121. https:// doi.org/10.22490/24629448.1735

Zupic, I., \& Čater, T. (2015) 'Bibliometric Methods in Management and Organization', Organizational Research Methods, 18(3), 429-472. https://doi. org/10.1177/1094428114562629 